\title{
Remarks on Lorentz symmetric spaces
}

\author{
Abdelghani Zeghib
}

\begin{abstract}
We consider homogeneous Lorentz spaces of dimension at least 3. We prove that if such a space has 'big' isotropy (that is, a non-precompact and irreducible isotropy group), then this space must have constant sectional curvature. As a corollary, we obtain a new direct proof of the fact that irreducible Lorentz symmetric spaces have constant curvature, which was known via (algebraic) classification.
\end{abstract}

In [CLPTV91], Cahen et al. used the following theorem.

Theorem 1. Let $(M, g)$ be an irreducible Lorentz symmetric space of dimension $\geqslant 3$, then it has constant sectional curvature.

To prove this, Cahen et al. examined the Berger list of irreducible symmetric pseudo-Riemannian spaces, and checked that the Lorentz signature of the metric implies constant sectional curvature. Afterwards Cahen et al. remarked that 'it would be certainly worthwhile to have a direct proof'. In [BI93], Bérard-Bergery and Ikemakhen also remarked that a direct proof of this fact would be interesting.

In this note we give a direct proof. Our result is as follows.

Theorem 2. Let $(M, g)$ be an irreducible homogeneous Lorentz space of dimension $\geqslant 3$, with non-precompact and irreducible isotropy group, then it has constant sectional curvature.

By non-precompact isotropy group, we mean that the closure of its image under the derivative representation (on the tangent space at its fixed point) is not compact (inside the orthogonal group $\mathrm{O}(1, n-1), n=\operatorname{dim} M)$.

Our proof here is of geometrico-dynamical flavour, in relation with [Zeg99], using the noncompactness of the isotropy group. In fact, we have known of this direct proof for a long time, but it was the remark in [CLPTV91] which convinced us of the interest.

Based on the 'almost definition' of symmetric spaces, 'the isotropy contains the holonomy', a direct algebraic approach to Theorem 2 is also available (see, for instance, [DO01, BZ]). We give here a classification-free geometric proof. Witte [Wit01] proved (but did not state) a variation on Theorem 2 with a non-geometric ergodic theory argument.

To start with, note the following rigidity.

Proposition 3. Let $M$ be a Lorentz manifold such that, for any $x \in M$, there are $H^{1}, \ldots, H^{d}$ totally geodesic lightlike hypersurfaces containing $x$, such that their characteristic directions $\left(T_{x} H^{1}\right)^{\perp}, \ldots,\left(T_{x} H^{d}\right)^{\perp}$ generate $T_{x} M$. Then $M$ has constant curvature.

Received 16 April 2003, accepted in final form 20 June 2003, published online 15 October 2004.

2000 Mathematics Subject Classification 53B30, 22F30.

Keywords: Lorentz symmetric, geodesic hypersurface, non-compact isotropy.

This journal is (C) Foundation Compositio Mathematica 2004. 


\section{A. ZEGHIB}

Proof. Fix $x \in M$, denote $T_{x} H^{i}$ by $B^{i}$, and choose $b^{i}$ an isotropic vector such that $B^{i}=\left(b^{i}\right)^{\perp}$.

For $u \in T_{x} M$, denote by $A_{u}$ the curvature operator $A_{u}: v \in T_{x} M \rightarrow R(u, v) u \in T_{x} M$. Then, for $u \in B^{i}, A_{u}$ preserves $B^{i}$ (since totally geodesic submanifolds are 'invariant' by the curvature operator). Moreover, $A_{u}\left(b^{i}\right)$ is collinear to $b^{i}$ (for $u \in B^{i}$ ). Indeed $\left\langle A_{u}\left(b^{i}\right), v\right\rangle=\left\langle A_{u}(v), b^{i}\right\rangle$. The last quantity equals zero if $v \in B^{i}$, since $A_{u}(v) \in B^{i}$ and, hence, $A_{u}\left(b^{i}\right) \in\left(B^{i}\right)^{\perp}=\mathbf{R} b^{i}$.

Choose $e_{i}$ a unit director vector of $\bigcap_{j \neq i} B^{j}$, and consider $A_{e_{i}}$. Since $e_{i} \in B^{j}$, for $j \neq i$, there is $\lambda_{i, j}$ such that $A_{e_{i}} b^{j}=\lambda_{i, j} b^{j}$ (for $i \neq j$ ).

Since $A_{e_{i}}$ is symmetric, $\lambda_{i, j}\left\langle b^{j}, b^{k}\right\rangle=\left\langle A_{e_{i}} b^{j}, b^{k}\right\rangle=\left\langle A_{e_{i}} b^{k}, b^{j}\right\rangle=\lambda_{i, k}\left\langle b^{j}, b^{k}\right\rangle$, and we have $\lambda_{i, j}=$ $\lambda_{i, k}$ (for $j \neq k,\left\langle b^{j}, b^{k}\right\rangle \neq 0$, because both $b^{j}$ and $b^{k}$ are isotropic). Write $\lambda_{i}=\lambda_{i, j}$. Thus, the sectional curvature of any non-degenerate plane which contains $e_{i}$ equals $\lambda_{i}$. From this, we infer that $\lambda_{1}=\cdots=\lambda_{n}$ (to see this, consider 2-planes generated by two vectors $e_{i}$ and $e_{j}$ ). One may use standard algebraic manipulations to show that all the 2-planes in $T_{x} M$ have the same sectional curvature. In order to deduce from this that the constant is the same everywhere in $M$, we use the following fact. This will which complete the proof of the theorem.

FACT 4 (Weak Schur's lemma, see for instance [KN63, p. 202]). If a pseudo-Riemannian manifold of dimension $\geqslant 3$ has constant curvature at each of its points, then this constant is the same everywhere on the manifold; that is to say, the manifold has constant curvature.

Remark 5. We called this fact the weak Schur's lemma, because the most well-known version of Schur's lemma concerns Ricci curvature; that is, if the Ricci curvature is everywhere proportional to the metric, then the proportionality constant is the same for all the points of the pseudo-Riemannian manifold, which is in fact, up to a normalization constant, the scalar curvature. In other words, the manifold is an Einstein manifold.

Now, we have the following.

FACT 6. Let $M$ be as in Theorem 2, then it contains a totally geodesic lightlike hypersurface (and, hence, by homogeneity, such a hypersurface through each of its points).

Proof. Consider $f \in \operatorname{Iso}_{x_{0}}$ (the isotropy group of a point $x_{0} \in M$ ) and its graph $\operatorname{Graph}(f) \subset$ $M \times M$. It is an isotropic totally geodesic $d$-dimensional submanifold of $M \times M$, when equipped with the metric $g \oplus(-g)(d=\operatorname{dim} M)$. If $f_{n} \in \mathrm{Iso}_{x_{0}}$ is a sequence tending to infinity, $\operatorname{Graph}\left(f_{n}\right)$ converges to $H^{\prime}$, an $n$-dimensional isotropic totally geodesic submanifold, which is no longer a graph. Its intersection with $\left\{x_{0}\right\} \times M$ is non-trivial, but has at most dimension 1 , because it is isotropic and $M$ is Lorentzian. Therefore, the projection $H$ of $H^{\prime}$ is a totally geodesic hypersurface in $M \times\left\{x_{0}\right\}$, which can easily be seen to be lightlike.

Proof of Theorem 2. The isotropic directions corresponding to all lightlike totally geodesic hypersurfaces containing $x_{0}$, generate a subspace of $T_{x_{0}} M$ invariant by $I s o_{x_{0}}$. In the irreducible case, this is the whole space and this allows one to apply Proposition 3 to conclude.

For Lorentz symmetric spaces, we have the following general fact which completes the proof of Theorem 1.

FACT 7. Let $M$ be a Lorentz symmetric space, then it contains a totally geodesic lightlike hypersurface (and, hence, such a hypersurface through each of its points).

Proof. One can restrict the analysis to the case where $M$ is weakly irreducible; that is to say, the holonomy does not preserve any non-degenerate space. Indeed, if a non-degenerate subspace is invariant by the holonomy, then it gives a parallel decomposition of the manifold together with its orthogonal space, which reduces the problem to the non-decomposable case. 


\section{REMARKS ON LORENTZ SYMMETRIC SPACES}

If $M$ is weakly irreducible, but non-irreducible, then, by definition, it has a parallel direction field or, equivalently, a parallel lightlike hyperplane field. It is integrable, with totally geodesic leaves, which proves our claim in this case.

In the other case, that is when $M$ is irreducible, let $H$ be the isotropy group of a point $x_{0}$. Then $H$ cannot be precompact by the following argument. Suppose that $H$ is precompact. Then the holonomy group $\mathrm{Hol}_{x_{0}}$ is precompact, and hence preserves a Riemannian scalar product on $T_{x_{0}} M$. As it also fixes the Lorentzian product, it preserves non-trivial eigenspaces of the matrix representing the Lorentzian product with respect to an orthonormal basis, contradicting the irreducibility of $M$.

\section{Some comments and questions}

Let us mention here some related questions.

1) The statement of Proposition 3 can be adapted to the general pseudo-Riemannian case and, in particular, to the Riemannian case. This implies the known fact that, in a irreducible symmetric Riemannian manifold of non-constant curvature, (proper) totally geodesic submanifolds have at least codimension 2 .

2) Another striking fact in Lorentz geometry is that (assuming the dimension $\geqslant 3$ ), if the curvature is bounded at some point, then it is constant (at this point). This is implicit in the proof of Theorem 1.3.1 of [Wol64], and explicit, for instance, in [BP84]. The reason for this is that the sectional curvature at a fixed point gives rise to a mereomorphic function, which cannot be bounded unless constant. This function is invariant by the isotropy group. This fact is specific to Lorentz (not necessarily symmetric) manifolds. Now, in the general pseudo-Riemannian case, the idea is to ask whether a 'big' isotropy can leave a non-trivial mereomorphic function invariant. This would lead to results similar to Theorem 2 for pseudo-Riemannian manifolds, assuming a sufficiently large isotropy group.

\section{ACKNOWLEDGEMENT}

I would like to thank the referee for his valuable suggestions and remarks.

\section{REFERENCES}

BP84 J. Beem and P. Parker, Values of pseudo-Riemannian sectional curvature, Comment. Math. Helv. 59 (1984), 319-331.

BI93 L. Bérard-Bergery and A. Ikemakhen, On the holonomy of Lorentzian manifolds, in Differential geometry: geometry in mathematical physics and related topics. Proc. Sympos. Pure Math. 54 (1993), 27-40.

BZ C. Boubel and A. Zeghib, Isometric actions of Lie subgroups of the Moebius group, Nonlinearity, to appear.

CLPTV91 M. Cahen, J. Leroy, M. Parker, F. Tricerri and L. Vanhecke, Lorentz manifolds modeled on a Lorentz symmetric space, J. Geom. Phys. 7 (1991), 571-581.

DO01 A. Di Scala and C. Olmos, The geometry of homogeneous submanifolds of hyperbolic space, Math. Z. 237 (2001), 199-209.

KN63 S. Kobayashi and K. Nomizu, Foundations of differential geometry, vol I (Interscience, New York, 1963).

Kow96 N. Kowalsky, Noncompact simple automorphism groups of Lorentz manifolds and other geometric manifolds, Ann. of Math. (2) 144 (1996), 611-640. 


\section{REMARKS ON LORENTZ SYMMETRIC SPACES}

Mos55 G. Mostow, Some new decomposition theorems for semi-simple groups, Mem. Amer. Math. Soc. 1955 (1955), 31-54.

Wit01 D. Witte, Homogeneous Lorentz manifolds with simple isometry group, Beiträge Algebra Geom. 42 (2001), 451-461.

Wol64 J. Wolf, Isotropic manifolds of constant curvature, Comm. Math. Helv. 39 (1964), 21-64.

Zeg99 A. Zeghib, Isometry groups and geodesic foliations of Lorentz manifolds. Part I: foundations of Lorentz dynamics, GAFA, Geom. Funct. Anal. 9 (1999), 775-822.

Abdelghani Zeghib zeghib@umpa.ens-lyon.fr

CNRS, UMPA, École Normale Supérieure de Lyon, 46, allée d'Italie, 69364 Lyon cedex 07, France 